\title{
Erythematous Indurated Nodule on the Forehead
}

\author{
Karan Pandher, MD; Felipe B. Cerci, MD, MSc; Stanislav N. Tolkachjov, MD
}

\section{Eligible for 1 MOC SA Credit From the ABD}

This Photo Challenge in our print edition is eligible for 1 self-assessment credit for Maintenance of Certification from the American Board of Dermatology (ABD). After completing this activity, diplomates can visit the ABD website (http://www.abderm.org) to self-report the credits under the activity title "Cutis Photo Challenge." You may report the credit after each activity is completed or after accumulating multiple credits.

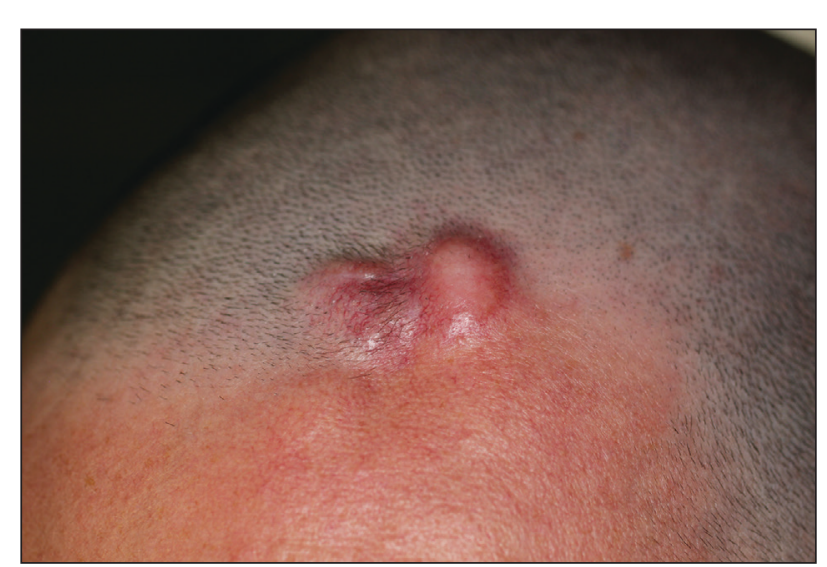

A 39-year-old man presented with an enlarging asymptomatic nodule on the forehead of more than 3 years' duration. Physical examination revealed a $3.4 \times 2.3-\mathrm{cm}$, indurated, firm, erythematous nodule on the frontotemporal scalp. The patient denied any history of trauma to the area.

\section{WHAT'S YOUR DIAGNOSIS?}
a. dermatofibrosarcoma protuberans
b. epidermoid cyst
c. lipoma
d. malignant peripheral nerve sheath tumor
e. plexiform fibrohistiocytic tumor

Dr. Pandher is from Chicago Medical School, Rosalind Franklin University of Medicine and Science, Illinois. Dr. Cerci is from the Postgraduate Program, Internal Medicine and Health Sciences, Universidade Federal do Paraná, Curitiba, Brazil, and Clínica Cepelle, Curitiba. Dr. Tolkachjov is from Epiphany Dermatology, Lewisville, Texas.

The authors report no conflict of interest.

Correspondence: Stanislav N. Tolkachjov, MD, Epiphany Dermatology, 1640 FM 544, Ste 3, Lewisville, TX 75056 (stan.tolkachjov@gmail.com). doi:10.12788/cutis.0434 


\section{THE DIAGNOSIS: Dermatofibrosarcoma Protuberans}

$\mid$ istopathologic examination showed a dermal tumor composed of spindle cells in a storiform arrangement (Figure 1). Immunohistochemistry demonstrated positive CD34 staining of the tumoral cells (Figure 2). Clinical review, histopathologic examination, and immunohistochemistry confirmed a diagnosis of dermatofibrosarcoma protuberans (DFSP). The patient underwent Mohs micrographic surgery (MMS) with clear margins after 3 stages, followed by repair with a rotation flap. No evidence of recurrence was found at 4-year follow-up.

Dermatofibrosarcoma protuberans is a rare low-grade sarcoma of fibroblast origin with an annual incidence of 0.8 to 5 cases per million individuals. ${ }^{1}$ It typically presents in patients aged 30 to 50 years on the trunk, scalp, or proximal extremities as an asymptomatic, flesh-colored, erythematous or brown, indurated plaque or nodule. ${ }^{2}$ Due to its variable presentation, these lesions often may be misdiagnosed as lipomas or epidermoid cysts, preventing proper targeted treatment. Therefore, suspicious enlarging indurated nodules require a lower threshold for biopsy. ${ }^{1}$

A definitive diagnosis of DFSP is achieved after a biopsy and histopathologic evaluation. Hematoxylin and eosin staining typically shows diffuse infiltration of the dermis and the subcutaneous fat by densely packed, cytologic, relatively uniform, spindle-shaped tumor cells arranged in a characteristic storiform shape. Tumor cells are spread along the septae of the subcutaneous fatty tissue. ${ }^{3}$ Immunohistochemistry is characterized by positive CD34 and negative factor XIIIa, with rare exceptions.

The differential diagnosis includes lipoma, epidermoid cyst, plexiform fibrohistiocytic tumor, and malignant peripheral nerve sheath tumor. ${ }^{3}$ Positive CD34 immunostaining, negative S-100 staining, and a storiform pattern of spindle cells can assist in differentiating DFSP from these possible differential diagnoses; lesions of these other entities are characterized by different pathologic findings. Lipomas are composed of fat tissue, epidermoid cysts have epithelial-lined cysts filled with keratin, plexiform fibrohistiocytic tumors have plexiform rays of fibrous tissue extending into fat with negative CD34 staining, and malignant peripheral nerve sheath tumors have fleshy variegated masses involving the peripheral nerve trunks with partial S-100 staining. ${ }^{4-7}$ Additional evaluation to confirm DFSP can be accomplished by analysis of tumor samples by fluorescence in situ hybridization or reverse transcriptase-polymerase chain reaction to detect chromosomal translocations and fusion gene transcripts, as chromosomal translocations may be found in more than $90 \%$ of cases. ${ }^{3}$

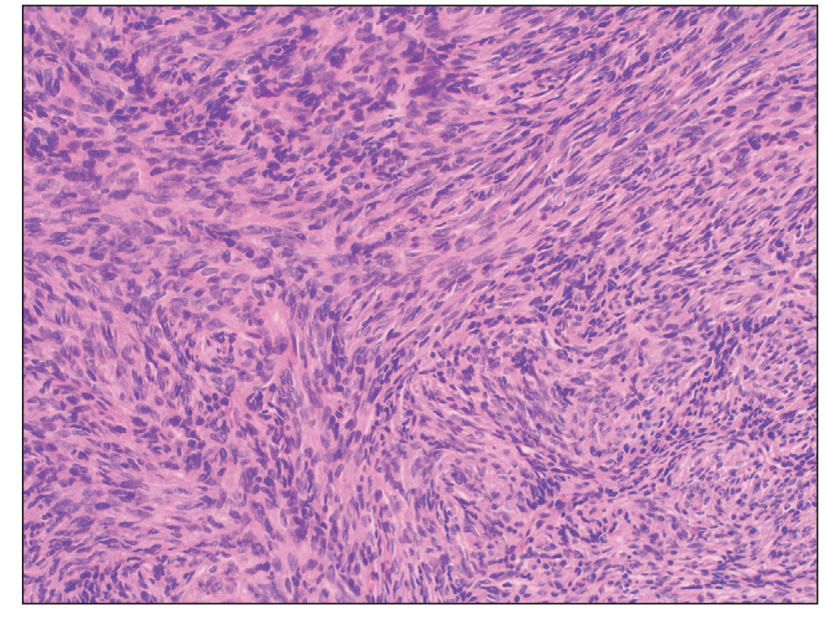

FIGURE 1. Histopathologic examination showed a dermal tumor composed of spindle cells in a storiform arrangement (H\&E, original magnification $\times 200$ ).

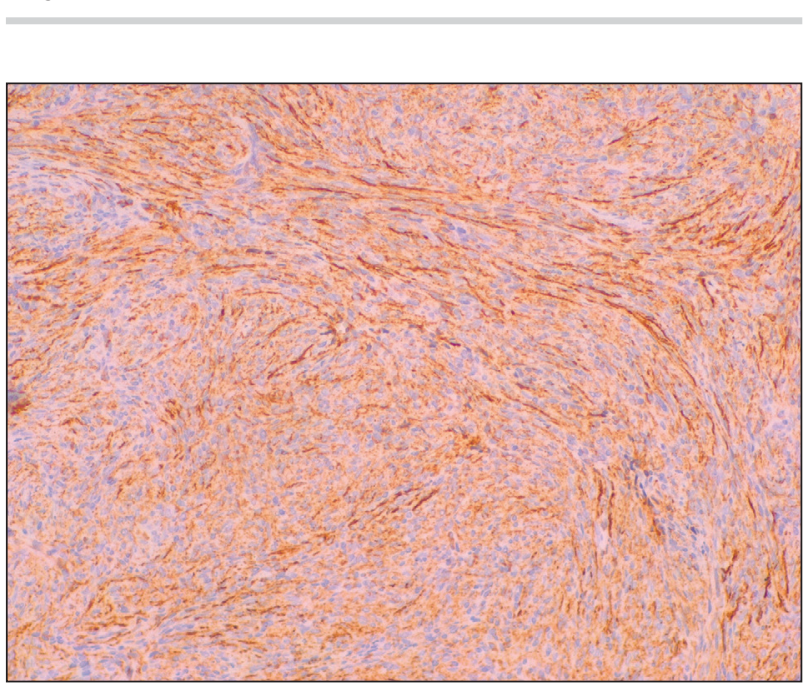

FIGURE 2. Immunohistochemistry showed positive CD34 staining of the tumoral cells (original magnification $\times 100$ ).

Early diagnosis of DFSP is beneficial, as it can help prevent recurrence as well as metastasis. Studies have attempted to document the risk for recurrence as well as metastasis based on characteristic features and treatment strategies of DFSP. In a study of 186 patients, 3 had metastatic disease to the lungs, the most common site of metastasis. ${ }^{8}$ These 3 patients had fibrosarcomatous transformation within DFSP, emphasizing the importance of detailing this finding early in the diagnosis, as it was characterized by a higher degree of cellularity, cytologic atypia, mitotic activity, and negative CD34 
immunostaining. ${ }^{9}$ In patients with suspected metastasis, lymph node ultrasonography, chest radiography, and computed tomography may be utilized. ${ }^{3}$

When treating DFSP, the goal is complete removal of the tumor with clear margins. Mohs micrographic surgery, modified MMS, and wide local excision (WLE) with 2- to 4-cm margins are appropriate treatment options, though MMS is the treatment of choice. A study comparing MMS and WLE demonstrated 3\% and $30.8 \%$ recurrence rates, respectively. ${ }^{8}$ In MMS, complete margin evaluation on microscopy is performed after each stage to ensure negative surgical margins. The presence of positive surgical margins elicits continued resection until the margins are clear. ${ }^{10,11}$

Other treatment modalities may be considered for patients with DFSP. Molecular therapy with imatinib, an oral tyrosine kinase inhibitor targeting platelet-derived growth factor-regulated expression, can be utilized for inoperable tumors; however, additional clinical trials are required to ensure efficacy. ${ }^{3}$ Surgical removal of the possible remaining tumor is still recommended after molecular therapy. Radiotherapy is an additional method of treatment that may be used for inoperable tumors. ${ }^{3}$

Dermatofibrosarcoma protuberans is a rare lowgrade sarcoma of fibroblast origin that typically does not metastasize but often has notable subclinical extension and recurrence. Differentiating DFSP from other tumors often may be difficult. A protuberant, flesh-colored, slowgrowing, and asymptomatic lesion often may be confused with lipomas or epidermoid cysts; therefore, biopsies with immunohistostaining for suspicious lesions is required. ${ }^{12}$ Mohs micrographic surgery has evolved as the treatment of choice for this tumor, though WLE and new targeted molecular therapies still are considered. Proper diagnosis and treatment of DFSP is paramount in preventing future morbidity.

\section{REFERENCES}

1. Benoit A, Aycock J, Milam D, et al. Dermatofibrosarcoma protuberans of the forehead with extensive subclinical spread. Dermatol Surg. 2016;42:261-264. doi:10.1097/DSS.0000000000000604

2. Khachemoune A, Barkoe D, Braun M, et al. Dermatofibrosarcoma protuberans of the forehead and scalp with involvement of the outer calvarial plate: multistaged repair with the use of skin expanders. Dermatol Surg. 2005;31:115-119. doi:10.1111/j.1524-4725.2005.31021

3. Saiag P, Grob J-J, Lebbe C, et al. Diagnosis and treatment of dermatofibrosarcoma protuberans. European consensus-based interdisciplinary guideline. Eur J Cancer. 2015;51:2604-2608. doi:10.1016/j.ejca.2015.06.108

4. Charifa A, Badri T. Lipomas, pathology. StatPearls. StatPearls Publishing; 2020.

5. Zito PM, Scharf R. Cyst, epidermoid (sebaceous cyst). StatPearls. StatPearls Publishing; 2020.

6. Taher A, Pushpanathan C. Plexiform fibrohistiocytic tumor: a brief review. Arch Pathol Lab Med. 2007;131:1135-1138. doi:10.5858 /2007-131-1135-PFTABR

7. Rodriguez FJ, Folpe AL, Giannini C, et al. Pathology of peripheral nerve sheath tumors: diagnostic overview and update on selected diagnostic problems. Acta Neuropathol. 2012;123:295-319. doi:10.1007 /s00401-012-0954-z

8. Lowe GC, Onajin O, Baum CL, et al. A comparison of Mohs micrographic surgery and wide local excision for treatment of dermatofibrosarcoma protuberans with long-term follow-up: the Mayo Clinic experience. Dermatol Surg. 2017;43:98-106. doi:10.1097/DSS.0000000000000910

9. Rouhani P, Fletcher CDM, Devesa SS, et al. Cutaneous soft tissue sarcoma incidence patterns in the U.S.: an analysis of 12,114 cases. Cancer. 2008;113:616-627. doi:10.1002/cncr.23571

10. Ratner D, Thomas CO, Johnson TM, et al. Mohs micrographic surgery for the treatment of dermatofibrosarcoma protuberans. results of a multiinstitutional series with an analysis of the extent of microscopic spread. J Am Acad Dermatol. 1997;37:600-613. doi:10.1016/s0190 -9622(97)70179-8

11. Buck DW, Kim JYS, Alam M, et al. Multidisciplinary approach to the management of dermatofibrosarcoma protuberans. J Am Acad Dermatol. 2012;67:861-866. doi:10.1016/j.jaad.2012.01.039

12. Shih P-Y, Chen C-H, Kuo T-T, et al. Deep dermatofibrosarcoma protuberans: a pitfall in the ultrasonographic diagnosis of lipoma -like subcutaneous lesions. Dermatologica Sinica. 2010;28:32-35. doi:10.1016/S1027-8117(10)60005-5 